\title{
UNIVERSIDAD NACIONAL: REACCIONES Y EFECTOS DEL HOSTIGAMIENTO SEXUAL EN LA POBLACIÓN ESTUDIANTIL ENEL 2008
}

\section{UNIVERSIDAD NACIONAL: SEXUAL HARRASMENT'S RESPONSES AND EFFECTS IN THE UNIVERSITY STUDENT IN 2008}

\author{
Zaira Carvajal Orlich* \\ Patricia Delvó Gutiérrez**
}

RESUMEN

En este artículo se presentan los resultados referentes a los efectos y reacciones que indicaron los estudiantes que respondieron positivamente haber sufrido alguna manifestación de hostigamiento sexual durante su permanencia en la Universidad Nacional. Los mismos se recopilaron en una encuesta realizada entre octubre $y$ noviembre de 2008, a una muestra de estudiantes de la UNA, donde se investigaron además temas como la prevalencia, las manifestaciones y la percepción que la población estudiada tiene sobre el ambiente homofóbico en la UNA.

PALABRAS CLAVE: COSTA RICA * UNIVERSIDAD NACIONAL * ACOSO SEXUAL * ESTUDIANTES $*$ COMPORTAMIENTO $*$ CONSECUENCIAS SOCIALES $*$ ADMINISTRACIÓN UNIVERSITARIA

\section{ABSTRACT}

This article presents the results about effects and reactions which were pointed of the students that suffered at least one of the manifestations of sexual harassment during the time they stay at the Universidad Nacional. This information was collected in a survey made in 2008, between October and November, to UNA's student sample. The subjects in this survey were prevalence, manifestations and the perception of the homofobic environment in the UNA.

KEYWORDS: COSTA RICA * UNIVERSIDAD NACIONAL * SEXUAL HARASSMENT * STUDENTS $*$ BEHAVIOUR $*$ SOCIAL IMPLICATIONS $*$ COLLEGE MANAGEMENT

* Instituto de Estudios de la Mujer de la Universidad Nacional

zcarvaja@una.ac.cr
** Instituto de Estudios de la Mujer de la Universidad Nacional. Docente de la Universidad de Costa Rica. pdelvo@hotmail.com 


\section{INTRODUCCIÓN}

En esta investigación el concepto de hostigamiento sexual se definió como lo establece la Ley contra el hostigamiento sexual en el empleo y la docencia (Ley 7476 de la República de Costa Rica), que dice: "Es toda conducta sexual indeseada por quien la recibe". Asimismo, en el artículo 5 de dicha ley se señala la responsabilidad patronal de mantener políticas de prevención. En este sentido, el 5 de noviembre de 1996 se aprobó en la Universidad Nacional (UNA) el Reglamento para prevenir, investigar y sancionar el hostigamiento sexual, el cual contempla un programa institucional de prevención y la realización de investigaciones en esta materia.

En este artículo se dan a conocer los resultados sobre los efectos ${ }^{1} y$ reacciones ${ }^{2}$ que indicaron los estudiantes que señalaron haber sufrido por lo menos una manifestación de hostigamiento sexual durante su permanencia en la Universidad Nacional. Los mismos forman parte de la información recolectada mediante una encuesta aplicada a una muestra de 1144 estudiantes, durante los meses de octubre $y$ noviembre de 2008. Además, se investigó sobre prevalencia y manifestaciones del hostigamiento sexual, percepción de las y los estudiantes sobre la existencia de un ambiente homofóbico $y$ lésbico en la UNA, entre otros.

La muestra seleccionada se conformó de 79 grupos, con representación de todas las carreras que imparte la UNA, en todas sus sedes.

El hostigamiento sexual, cualquiera que sea la forma en que se manifieste, causa daño en el estado de bienestar personal de quienes lo reciben, lo cual incluye aspectos emocionales, físicos, sociales y en el caso particular de un centro de enseñanza, el mismo afecta el rendimiento académico. Esta forma de violencia constituye una violación a los derechos huma-

1 Comprende los daños emocionales, conductuales, económicos y de estudio que causó a las personas afectadas por una o más manifestaciones del hostigamiento sexual, de manera inmediata y a más largo plazo.

2 Acción que realiza una persona ante una conducta que fue dirigida contra ella. nos, porque limita las condiciones de estudio y de trabajo.

Si bien es cierto, cualquier persona, mujer u hombre, puede ser víctima de violencia, también lo es el hecho de que, en las sociedades patriarcales el sexismo es la causa para que se cometan y legitimen una serie de abusos y agresiones contra las mujeres, sólo por el hecho de serlo.

La violencia que se comete contra las mujeres, la violencia doméstica en sus diferentes manifestaciones (física y psicológica), en relación de pareja, violación e incesto, así como el abuso sexual y el hostigamiento sexual, forman parte de una problemática social que mantiene estrecha relación con el ejercicio abusivo del poder. A su vez, en el marco de una política sexual discriminatoria y sistemática es utilizada como un mecanismo de opresión y subordinación contra las mujeres.

Existe poca información en relación con el hostigamiento sexual en el ámbito estudiantil universitario, por lo que un estudio como este, con resultados generalizables a la población estudiantil de la Universidad Nacional, es importante para orientar acciones de prevención y promover la denuncia y la sanción correspondiente.

\section{ACERCAMIENTO TEÓRICO}

A continuación se plantean algunos conceptos que dentro de la teoría de género son importantes para interpretar los resultados obtenidos de esta investigación.

\section{PATRIARCADO}

Según Victoria Sau, el patriarcado es

... una toma de poder histórica por parte de los hombres sobre las mujeres cuyo agente ocasional fue de orden biológico, si bien elevado este a la categoría política y económica. Dicha toma de poder pasa forzosamente por el sometimiento de las mujeres a la maternidad, la represión de la sexualidad femenina y la apropiación de la fuerza de trabajo total del grupo 
dominado, del cual su primer pero no único producto son los hijos (Sau, 1989: 239-9).

El patriarcado según Adrianne Rich

... consiste en el poder de los padres: un sistema familiar y social, ideológico y político con el que los hombres - a través de la fuerza, la presión directa, los rituales, la tradición, la ley o el lenguaje, las costumbres, la etiqueta, la educación y la división del trabajo- determinan cuál es o no es el papel que las mujeres deben interpretar con el fin de estar en toda circunstancia sometidas al varón (Rich, 1978: 22).

Este sistema crea desigualdades sociales $y$ de poder entre los sexos, que se mantiene $y$ perpetua, precisamente, por esas injusticias de poder. Las sociedades patriarcales son androcéntricas $^{3}$, lo que significa que el hombre y lo masculino son tomados como modelo de lo humano. En correspondencia con la ideología patriarcal los hombres tienen el mandato de dominar y controlar los bienes, la naturale$\mathrm{za}$, los medios de producción, las mujeres, los niños y las niñas y también a algunos hombres. En tanto las mujeres, tienen el mandato de ser sumisas, obedientes y servidoras de los hombres. Se construye entonces un marco de relaciones desiguales de poder que resulta propicio para la génesis de la violencia contra las mujeres.

Como consecuencia de ello, que los hombres digan bromas, chistes, tengan acercamientos $y$ toqueteos contra las mujeres, es percibido como "algo normal", se sienten legitimados. Por su parte las mujeres (aunque les disguste) creen que tienen que soportar el hostigamiento sexual, como parte del contrato de trabajo o de estudio, ya que si muestran resistencia y enojo, podrían ser despedidas o reprobadas

3 Androcentrismo proviene del griego Andros (hombre) $y$ define la mirada masculina en el centro del Universo, como medida de todas las cosas y representación global de la humanidad, ocultando otras realidades, entre ellas la de las mujeres. de un curso, precisamente por no aceptar los requerimientos de su jefe o profesor.

En cualquier espacio de la cotidianidad es observable que "los sujetos" son los hombres y "los objetos" las mujeres. Esta dicotomía es construida socialmente $y$ basada en el sexo, con base en una serie de mitificaciones que coloca a unos en la esfera del poder y a las otras en la subordinación.

\section{JERARQUÍAS}

La idea de jerarquía ${ }^{4}$ enlaza la estructuración de un conjunto de diferentes rangos atendiendo criterios de derivación formal y material. Una metáfora útil podría ser la de una pirámide dividida en escalones. El principio de jerarquía es el garante de la estructura, de la unidad del ordenamiento.

Una característica de las sociedades patriarcales es que son profundamente jerarquizadas, por situación económica, etnia, religión, sexo y orientación sexual, estableciéndose rangos de superioridad e inferioridad entre personas y grupos. En cuanto al sexo, las desigualdades sociales entre mujeres y hombres, históricamente, han sido vistas como algo natural, aceptándose como un hecho inmodificable la dominación de lo masculino sobre lo femenino. De manera concomitante, el poder hegemónico de los hombres se ha normalizado con desventaja para las mujeres.

Los estudios más conocidos sobre el hostigamiento sexual refieren que hay dos tipos de jerarquías, las cuales favorecen la ventaja de poder que ostentan los hombres sobre las mujeres. Una de ellas está referida a la superioridad jerárquica laboral, en donde son los hombres quienes mayoritariamente están ubicados en posiciones de jefatura. La otra es la superioridad jerárquica por razones de género, en la cual los patrones ideológicos culturales promueven que ellos se coloquen en el centro, con rango de Se define por jerarquía la "organización por categorías o grados de importancia entre diversas personas o cosas", según la Real Academia Española (RAE).
} 
superioridad sobre el género femenino. En este contexto sus demandas no puedan ser cuestionadas ni rechazadas, como apunta García y Bedoya (1993: 41) “... a los hombres se les educa a que su deseo no puede ser cuestionado, por lo que es difícil que acepten su rechazo".

Un enfoque más actualizado sobre el hostigamiento sexual y en general, sobre los otros tipos de violencia que se ejercen contra las mujeres de todas las edades (incesto, abuso sexual, violencia en relaciones de pareja, violación, entre otros) se fundamenta en lo que Kate Millett (1968) denomina politica sexual. Esta autora argumenta que el sexo es una categoría social impregnada de política, entendido este concepto como los métodos o tácticas usados en la dirección de un Estado o gobierno y que ella amplía al conjunto de estratagemas destinadas a mantener el sistema patriarcal.

De manera que, el sistema patriarcal es una institución perpetuada mediante una serie de relaciones y compromisos estructurados de acuerdo con el poder, en virtud de los cuales las mujeres quedan bajo el control de los hombres, constituyendo de acuerdo con Millett, una política sexual aprobada $y$ sostenida por medio de procesos de socialización diferenciados por sexo. Estos procesos involucran al menos tres componentes:

a) Psicológico (relacionado con el temperamento que deben mostrar las personas según el sexo).

b) Sociológico (referido a los roles o papeles que deben ser desempeñados según el sexo).

c) Político (vinculado a la posición social o rango jerárquico de cada sexo).

En una sociedad patriarcal y jerarquizada se dan condiciones propicias para la violencia contra las mujeres, porque

Las creencias culturales que sostienen un sistema de dominio masculino contribuyen a hacer a mujeres $y$ niños [sic] vulnerables sexualmente (...). El hecho de que la urgencia sexual masculina es vista como predominante $y$ necesaria de ser satisfecha, le permite al hombre justificar conductas sexuales antisociales, tales como el abuso sexual (Finkelhor, 1985: 48).

Como problemática social vinculada a jerarquías, el hostigamiento sexual se oculta y mitifica, causando enormes perjuicios psicológicos, sociales y económicos a quienes lo enfrentan.

\section{HOSTIGAMIENTO SEXUAL}

Si bien no hay una única definición de este concepto, las que existen coinciden en que es una conducta no bienvenida por quien la recibe. En un resumen de las distintas definiciones sobre esta conducta, García y Bedoya (1993) tratan de recuperar los siguientes elementos:

a) Son conductas de tipo sexual, que se manifiestan de manera diversa.

b) Se presentan en una situación desigual de poder entre el perpetrador y quien la recibe, ya sea por la posición jerárquica dentro de un ambiente laboral o de estudio o por el status que le da su condición de género.

c) Tienen la intención de acosar, presionar, coaccionar e intimidar a una persona.

d) No bienvenidas ni solicitadas por quien las recibe, además de que involucran una agresión sexual.

El hostigamiento sexual se puede manifestar en diversas formas, desde insinuaciones enmascaradas de halagos (piropos), pasando por miradas, gestos lascivos y verbalización de deseo, chistes y comentarios de naturaleza sexual, despliegue de material pornográfico, roces corporales, besos, hasta proposiciones directas de mantener relaciones sexuales.

En la práctica de las relaciones cotidianas entre las personas son palpables como muchas de estas agresiones pasan como "naturales o normales", como algo que tiene que suceder entre el jefe $y$ la subalterna, el docente y la/el estudiante, entre compañeras y compañeros de trabajo o de estudio.

El hostigamiento sexual también les puede ocurrir a los hombres. Las estadísticas 
indican que el porcentaje es menor que el que les ocurre a las mujeres. Si un hombre es hostigado por una mujer, Amalia Rivera apunta que “... el varón al verse asediado por una mujer puede resultar un hecho atractivo $y$ digno de ser comentado, al ser una situación que socialmente puede reforzar su virilidad" (Rivera en Bedolla, 1993: 136). No obstante, algunos tipos de hombres, principalmente los que no corresponden con el estereotipo masculino $y$ que son percibidos como homosexuales, son hostigados sexualmente por otros hombres. Sobre este punto José Manuel Salas analiza que se recurre a la homofobia ${ }^{5}$ como un dispositivo de control (1996: 75).

También persiste la resistencia a reconocer el hostigamiento sexual como una violación a los derechos humanos, a pesar de que constituye un ejercicio abusivo del poder, donde la sexualidad es utilizada como un mecanismo para intimidar, con frecuencia acompañado de violencia al utilizarse métodos coercitivos, para vencer la resistencia de quien está recibiendo el hostigamiento.

El hostigamiento sexual puede ocurrir tanto en una relación laboral o de estudio y constituye una extralimitación de los roles asignados a los hombres. El mismo se presenta cuando se proyecta en las mujeres una serie de conductas sexuales, asociados a la imagen de objeto sexual que ideológicamente se les atribuye. Se espera que las mujeres, independientemente del lugar donde estén, sean seductoras, complacientes, receptoras pasivas de acercamientos corporales, toqueteos, bromas con contenido sexual, entre otros, que por lo demás hacen aparecer a las mujeres como las promotoras de relaciones sexualizadas. En concordancia con lo anterior, generalmente se exime a los hombres de responsabilidad, porque ellos son "tentados por las mujeres".

\section{EFECTOS DEL HOSTIGAMIENTO SEXUAL}

El daño que puede causar esta forma de violencia en quienes la sufren de manera directa

Según Salas, se entiende por homofobia el rechazo evidente - a veces violento- de todo lo que tenga que ver con la homosexualidad (femenina o masculina). es inconmensurable, sus efectos perduran en el tiempo, afectando el pleno ejercicio del derecho al estudio libre de violencia, la salud, la estabilidad económica y el desempeño académico, asî como las relaciones familiares y en general el bienestar personal.

Datos de otros estudios sobre los efectos del hostigamiento sexual referidos al ámbito laboral señalan que, conductas de este tipo, afectan de manera negativa el ambiente en el trabajo y la productividad ${ }^{6}$. En estos estudios se señala, además que, por constituir el hostigamiento sexual un riesgo para la seguridad y la salud, el sector empresarial tiene la responsabilidad de tomar medidas para reducirlo "al mínimo", al igual que hace con otras situaciones de riesgo en el trabajo.

Relacionando lo anterior con el ámbito educativo que compete a este estudio, se puede homologar la productividad con el rendimiento académico, que posibilita el resultado más importante, toda vez que las y los estudiantes se inscriben en un centro de estudios de educación superior, para obtener un grado universitario. De igual manera, el jerarca de la institución está obligado a tomar acciones y medidas contra las conductas de hostigamiento sexual, dado que obstaculizan la libertad individual, constituyen una discriminación en general contra las mujeres y por supuesto pueden incidir en una imagen institucional negativa. También hay que mencionar el costo material que ocasionan estas prácticas, que al existir deben ser atendidas.

\section{Algunos estudios indican que}

Los efectos que tiene el hostigamiento sexual sobre quienes lo reciben no son sólo económicos, se manifiestan además en intentos de suicidio por parte de la perjudicada, fuerte pérdida de capacidad para volver a relacionarse con los demás, dificultad para establecer vínculos afectivos, depresiones recurrentes, dependencia de fármacos, $y$ otros tales como confusión, tres elementos: la calidad del proceso de producción, la calidad del producto y la calidad de vida de las y los trabajadores. 
bochorno, coraje y un gran sentido de degradación personal (Vicente, 1994: 5).

De manera análoga, en la tesis de posgrado de una de las autoras de este artículo (Carvajal, 2004: 74-89), se incluyeron varios testimonios de estudiantes que habían sufrido hostigamiento sexual, los cuales se consideran útiles para ilustrar cómo afecta a las y los estudiantes. Algunas de ellas habían desertado, definitiva o temporalmente, de la Universidad Nacional a consecuencia de los malestares causados por el hostigamiento sexual del que habían sido objeto. En palabras de algunas de las entrevistadas, así lo expresaron:

-Sí, yo me fui un tiempo porque estaba además, muy que, hastiada...

- Lo que hizo fue como desilusionarme y pese a todo yo seguí estudiando pero ya no llegué, empecé a bajar notas... no tengo la motivación por lo menos en este momento no siento el deseo de venir a estudiar a la Universidad.

-Tuve un examen de admisión bastante bueno... pero cuando eso sucedió se me quitaron las ganas $y$ dije yo me voy de aquí...
Igualmente en el aula se crea un ambiente de tensión, tanto para quienes sufren directamente la conducta de hostigamiento sexual, como para el grupo de compañeras y compañeros que la presencian. Se ha observado que el docente que hostiga busca alianzas para crear un ambiente hostil en contra de la o el estudiante, principalmente si esta (este) intenta o concreta una denuncia.

Directa o indirectamente el grupo de estudiantes, al igual que la persona afectada directamente, que ha sido involucrado en situaciones de este tipo enfrentan un dilema porque su objetivo es aprobar el curso y para ello dependen del docente que ha contaminado el ambiente con una conducta perversa.

Con base en los datos utilizados por Carvajal (2004) para realizar su estudio, en general se tiene que las mujeres más que los hombres expresaron el malestar que les causa el hostigamiento sexual.

\section{REACCIONES Y DENUNCIA}

Ante la acción de ser objeto de hostigamiento sexual la persona afectada tiene diversas reacciones, las cuales van desde minimizar el hecho hasta interponer una denuncia formal. En este estudio se incluyeron como reacciones ante el hostigamiento sexual las indicadas en el recuadro que sigue.

RECUADRO 1

REACCIONES CONSIDERADAS ANTE EL HOSTIGAMIENTO SEXUAL

\begin{tabular}{ll}
$\diamond$ Lo tomó a broma & $\diamond$ Se cambió de carrera \\
$\diamond$ No le dio importancia & $\diamond$ Se retiró, temporalmente de la UNA \\
$\diamond$ Lo rechazó & $\diamond$ Se lo comentó a alguien de su confianza \\
$\diamond$ No dijo nada, pero le molestó & $\diamond$ Se lo comentó a alguna autoridad universitaria de la UNA (do- \\
$\diamond$ No lo denunció por temor a represalias & cente, administrativo, etc.) \\
$\diamond$ Retiró o abandonó el curso & $\diamond$ Puso la denuncia una vez concluido el curso \\
\hline
\end{tabular}

Dado que por el Reglamento interno que regula esta materia en la UNA, existe un órgano institucional competente para la tramitación de cualquier denuncia sobre hostigamiento sexual, se consideró importante valorar, desde la óptica de la población encuestada cual fue la respuesta de las autoridades ante su denuncia (formalizada o no), así como las razones que tuvieron para no hacerla.

En el estudio ya citado de Carvajal (op. cit.) se recopilan las reacciones que expresaron 
algunas de las estudiantes entrevistadas, que habían sido objeto de hostigamiento sexual. En palabras de una esas estudiantes que interpuso la denuncia ante la Fiscalía contra el Hostigamiento Sexual de la UNA, tenemos:

- ... la que me ha motivado [a denunciar] es $\mathrm{C}$ [compañera], porque $\mathrm{C}$ es la que percibió las cosas, bueno más que yo y quizá me dio así como la sacudida... reaccioné, no puede dejar pasar así la situación, yo no sé si es que yo la dejé pasar tal vez inconscientemente la dejé pasar... yo lo veía casi como normal, como el hecho de vivir en un pueblo, yo soy de Turrialba, un pueblo donde también se ve mucha situación de esta, donde la mujer es sumisa y no tiene derecho a defenderse $y$ el hombre siempre tiene la razón, nunca pierde, entonces, no sé si por eso fue que no reaccioné...

Si bien es cierto cualquier persona, hombre o mujer, puede ser objeto de violencia, también lo es el hecho de que, en la sociedades patriarcales el sexismo es la causa para que se cometan y legitimen manifestaciones $y$ conductas violentas contra las mujeres, sólo por el hecho de ser mujeres. Estructural y políticamente el objetivo es mantener a las mujeres en estado de opresión y subordinación.

En relación con la denuncia, Carvajal (2004: 85) señala que:

Más mujeres que hombres lo comentaron con alguna persona de confianza y la denuncia aunque es más frecuente como reacción en las mujeres, es de por sí en términos porcentuales muy baja en relación con la alta prevalencia. En esto influye como uno de los factores limitantes, el temor a represalias, algo obvio que se desprende de las entrevistas a profundidad.

Como complemento a lo dicho anteriormente por la estudiante en la entrevista en profundidad, se agrega lo que opinó en relación con la denuncia:

Me da miedo la reacción del profesor cuando a él se le notifique (...) no a nivel de que vaya a tomar represalias contra todo el grupo, sino contra mí por eso ayer no pensé ir a clases, pero tampoco me interesa volver... (ibid, 74).

De manera que, la estudiante supone que en un proceso de denuncia ella llevaría la peor parte, porque culturalmente aprendió que los hombres son superiores a las mujeres. Sumado al hecho de que ella es alumna del profesor que la hostiga, asumiendo también que la palabra de él será más creíble que la de ella.

Es entendible el temor expresado por la estudiante, pero lo cierto es que las instancias de la institución, encargadas de atender estos procesos se encuentran sensibilizadas y capacitadas en materia de género, para entender que la problemática del hostigamiento sexual involucra una tríada entre poder, sexualidad y violencia.

\section{CARACTERIZACIÓN DEL ESTUDIO}

En el estudio cuantitativo para medir la prevalencia y manifestaciones del hostigamiento sexual de la población estudiantil en la UNA, matriculada durante el segundo ciclo lectivo del 2008 en las distintas sedes de esta universidad (no se consideraron la inter-universitaria de Alajuela ni el convenio con la Universidad de Costa Rica en Limón), se incluyeron además algunas preguntas sobre las reacciones de los y las estudiantes, la respuesta de las autoridades cuando se dio la denuncia del hecho y los efectos causados por el hostigamiento sexual.

\section{DELIMITACIÓN DEL OBJETO DE ESTUDIO}

Durante el segundo semestre de 2008, según los datos del Departamento de Registro de la UNA, el total de estudiantes que se matricularon ascendía a 10 984, de los cuales el $57,4 \%$ son mujeres (6307) y $42,6 \%$ hombres (4677).

Por razones prácticas, para tratar de reducir la posibilidad de tener estudiantes que estuvieran en dos grupos seleccionados, sólo se consideraron los cursos denominados "teóricos", dado que la mayoría de los cursos prácticos son laboratorios que tienen su complemento teórico. 
Para la selección de cursos/grupos se consideraron 2106 (equivale al 93,3\% del total de cursos/grupos que durante este semestre ascendió a 2264).

El estudio efectuado entre el 23 de octubre y el 15 de noviembre en la muestra de grupos seleccionados, consideró todas las sedes de la UNA; a saber: Omar Dengo, Benjamín Núñez, Sede Chorotega (Campus de Liberia y Nicoya) y Sede Brunca (Pérez Zeledón y Coto).

\section{INSTRUMENTO DE RECOLECCIÓN DE DATOS}

Para recolectar la información se usó un cuestionario autoadministrado que constaba de 5 páginas con 61 preguntas, agrupadas en 6 partes vinculadas a ejes temáticos. En la tabla siguiente se muestra el número de preguntas incluidas en cada eje temático.

TABLA 1

PARTE, EJES TEMÁTICOS Y NÚMERO DE PREGUNTAS INCLUIDAS EN EL CUESTIONARIO

\begin{tabular}{clc}
\hline PARTE & \multicolumn{1}{c}{ EJE TEMÁTICO } & PREGUNTAS \\
\hline I & Identificación & 5 \\
II & Prevalencia y manifestaciones & 23 \\
III & Reacciones personales y respuesta institucional & 3 \\
IV & Efectos & 23 \\
V & Ambiente homofóbico & 5 \\
VI & Campaña "Cero Tolerancia al Hostigamiento Sexual" & 2 \\
\hline
\end{tabular}

Las manifestaciones consideradas en el estudio son las que se detallan en la recuadro 2.

\section{RECUADRO 2}

\section{MANIFESTACIONES INDAGADAS EN EL CUESTIONARIO}

1. Exclamaciones o en gestos insinuantes, a su paso, que le disgustaron.

2. Comentarios o conversaciones sexuales, subidos de tono, no deseados.

3. Le han dicho obscenidades no deseadas en un aula, pasillo, laboratorio o trabajo de campo de la UNA.

4. Le han desnudado con la mirada y eso le disgustó.

5. Objeto de llamadas telefónicas, mensajes telefónicos, (mensajes de texto al celular) cartas o correos electrónicos no deseados de tipo sexual, por parte de alguien de la UNA.

6. Invitación a salir a tomar algo o a cenar con fines sexuales no deseados, que le disgustaron.

7. Comprometida/comprometido con personas de la UNA, a asistir a reuniones o fiestas de índole sexual no deseadas.

8. Tocada/tocado intencionalmente en cualquier parte de su cuerpo, no deseada por alguien de la UNA.

9. Acorralado o retenido, en contra de su voluntad por alguna persona de esta Universidad.

10. Choteado por su orientación sexual homosexual o lésbica por alguna persona de la UNA.

11. Le han sujetado su cuerpo, de forma particular, aprovechándose de las circunstancias en contra de sus deseos.

12. Contra sus deseos, alguien trató de averiguar sobre su vida sexual.

13. Uso de material pornográfico como revistas u otros por alguna persona de esta Universidad, sin ninguna necesidad.

14. Se mostró protectora/protector y luego le pidió favores sexuales.

15. Citada/citado en una oficina, laboratorio u otro sitio de la Universidad, innecesariamente, en horas en que generalmente no hay gente para hacerle proposiciones de tipo sexual. 


\section{RECUADRO 2 (CONTINUACIÓN) \\ MANIFESTACIONES INDAGADAS EN EL CUESTIONARIO}

16. Le hablaron de sus experiencias sexuales, sin que usted se lo haya pedido.

17. Presionada/presionado a tener contactos íntimos a cambio de darle por aprobado el curso, mejorar la nota, ayudarle con una beca u otros beneficios, o de lo contrario usted se vería afectada/afectado.

18. Han usado su nombre en mensajes, pintas con contenido sexual, en baños, paredes u otros en la UNA.

19. Le han discriminado por su masculinidad o feminidad diferente a la tradicionalmente existente.

20. Han utilizado en pintas o graffitis su nombre para evidenciar sus prácticas sexuales.

Previo a la realización del trabajo de campo, en el mes de setiembre se realizó una prueba del instrumento con 23 estudiantes que cursaban carreras de la Facultad de Ciencias Económicas de la Universidad de Costa Rica y que asistían a un curso de Estadística General I. Esta prueba permitió detectar algunos problemas y corregir la redacción de algunas de las preguntas.

\section{DISEÑO MUESTRAL}

La experiencia de la Fiscalía contra el Hostigamiento Sexual y el trabajo de concientización realizado por el Instituto de Estudios de la Mujer (IEM) de la Universidad Nacional, les ha permitido detectar diferencias al interior de las distintas facultades, sobre la presencia del hostigamiento sexual. Por esta razón se decidió agrupar las carreras en tres grandes estratos, para mejorar la precisión en la estimación de la prevalencia del hostigamiento sexual y garantizar la presencia en la muestra de carreras conocidas con alto porcentaje de este tipo de violencia.

En cada uno de los estratos definidos (alto, medio y bajo) se procedió a seleccionar 20 unidades de muestreo, las cuales estaban conformadas por uno o más grupos de estudiantes o cursos. Para la selección de estas unidades se utilizó muestreo sistemático proporcional al tamaño del grupo dentro de cada estrato definido (esto se hizo luego de ordenar los cursos dentro de las carreras) y cuando el número de estudiantes en el grupo seleccionado tenía reportada una matrícula inicial inferior a 20 estudiantes en los estratos alto y medio o de 17 en el estrato bajo, ese grupo se unió con el siguiente para formar una unidad de muestreo. Si el número de estudiantes era mayor, entonces constituía por sí solo una unidad de muestreo.

En el cuadro 1 se muestra la matrícula inicial de cada estrato, el número de cursos considerados y la matrícula promedio por curso, luego de haber realizado el procedimiento anterior.

CUADRO 1

UNIVERSIDAD NACIONAL: MATRÍCULA, CURSOS Y MATRÍCULA PROMEDIO POR CURSO PARA EL TOTAL Y LOS CURSOS SELECCIONADOS POR ESTRATO, NOVIEMBRE 2008

\begin{tabular}{ccccccc}
\hline \multirow{2}{*}{ ESTRATO } & \multicolumn{2}{c}{ MATRÍCULA POR CURSO } & \multicolumn{2}{c}{ CURSOS } & \multicolumn{2}{c}{ MATRÍCULA PROMEDIO } \\
\cline { 2 - 7 } & \multirow{2}{*}{ TOTAL } & \multirow{2}{*}{ SELECCIONADOS } & TOTAL & SELECCIONADOS & \multirow{2}{*}{ TOTAL } & \multirow{2}{*}{ SELECCIONADOS } \\
\hline TOTAL & 40876 & 1691 & 2106 & 79 & 19,4 & 21,4 \\
\hline Alto & 7725 & 558 & 409 & 25 & 18,9 & 22,3 \\
Medio & 26959 & 623 & 1330 & 25 & 20,3 & 24,9 \\
Bajo & 6192 & 510 & 367 & 29 & 16,9 & 17,6 \\
\hline
\end{tabular}


La justificación para seleccionar 20 unidades de muestreo por estrato, responde a la necesidad de simplificar el cálculo de la variabilidad entre los grupos seleccionados dentro de los estratos, pues esto permite tener 10 unidades pareadas.

\section{RECOLECCIÓN, PROCESAMIENTO Y TABULACIÓN} DE LOS DATOS

El cuestionario se aplicó a una muestra de 1144 estudiantes matriculados/as en 73 grupos y no en 79 como se planeó, porque se presentaron problemas de diversa índole que imposibilitaron recolectar la información en la población estudiantil de seis de los grupos seleccionados.

Con anterioridad a la realización del trabajo de campo, con el fin de estandarizar la recolección de los datos, se llevaron a cabo dos charlas para capacitar a las personas (estudiantes $y$ funcionarias) que iban a apoyar el proceso $y$ a cada participante se le entregó un instructivo.
Concluida la recolección de los datos se inició el proceso de codificación y digitación de la información, con base en un manual elaborado para tales efectos.

La base de datos se digitó en hojas en Excel, que se consolidaron luego en una sola base para proceder a su depuración. Para el procesamiento de los datos se usó principalmente la opción de "Tablas y Cuadros dinámicos" del Excel.

\section{RESULTADOS MÁS RELEVANTES \\ MAGNITUD DEL HOSTIGAMIENTO SEGÚN LAS PERSONAS HOSTIGADAS}

Con base en las 20 manifestaciones de hostigamiento sexual consideradas en este estudio, el 35,8\% de las y los estudiantes indicó haber recibido, al menos una de ellas, durante su permanencia como estudiantes de la Universidad Nacional.

\section{CUADRO 2}

UNIVERSIDAD NACIONAL. ESTUDIANTES QUE CON BASE EN MANIFESTACIONES HAN RECIBIDO HOSTIGAMIENTO SEXUAL SEGÚN SEXO EN VALORES ABSOLUTOS Y RELATIVOS, 2008

\begin{tabular}{lcccccc}
\hline \multirow{2}{*}{ CATEGORÍAS } & \multicolumn{3}{c}{ VALORES ABSOLUTOS } & \multicolumn{3}{c}{ VALORES RELATIVOS } \\
\cline { 2 - 7 } & TOTAL & HOMBRES & MUJERES & TOTAL & HOMBRES & MUJERES \\
\hline TOTAL ENCUESTADO ${ }^{1 /}$ & 1139 & 516 & 623 & 100,0 & 100,0 & 100,0 \\
Ha recibido hostigamiento sexual & 408 & 145 & 263 & $35,8 \%$ & $28,0 \%$ & $42,3 \%$ \\
No ha recibido hostigamiento sexual & 731 & 372 & 379 & $64,2 \%$ & $72,0 \%$ & $57,7 \%$ \\
\hline
\end{tabular}

1/ No incluye 5 estudiantes que no informaron su sexo.

El cuadro anterior muestra que el porcentaje de prevalencia del hostigamiento sexual se diferencia por sexo, por cada diez hombres hostigados hay 15 mujeres hostigadas. Estos datos confirman que la mujer socialmente es tratada como objeto sexual, —situación que la hace más vulnerable ante el problema-, lo que no es aplicable a los hombres, pues ellos son los sujetos y no objetos.

Estas diferencias confirman lo dicho por varias estudiosas de esta problemática, en cuanto a que el hostigamiento sexual es un mecanismo eficaz que se utiliza para ejercer dominio y opresión, cuya finalidad es mantener al género femenino en la categoría subordinada. Las mujeres por consiguiente estudian, en este caso, en un ambiente desventajoso y muchas veces hostil, que las afecta en el estado de bienestar personal y las priva del derecho a estudiar libres de violencia.

Además, conviene señalar que, las relaciones del ámbito académico deben estar basadas en el respeto mutuo, de manera que, no interfieran ni en el buen desempeño laboral ni tampoco en el rendimiento académico estudiantil. Porque al igual que lo refieren algunos estu- 
dios a nivel de empresa, el hostigamiento sexual acarrea pérdidas económicas, además de los daños en las personas afectadas (Vicente, 1995: 5).

\section{EFECTOS}

Como se indicó en el acápite correspondiente al acercamiento teórico, por razones ideológicas patriarcales, estructurales y políticas, el hostigamiento sexual se da mayoritariamente contra las mujeres. De igual manera, las mujeres más que los hombres, sienten malestar ante el hostigamiento sexual que se dirige contra ellas, porque sus efectos son acumulativos, un recordatorio constante de que es mujer y que por ello tiene un rango inferior en la estructura jerárquica de género, toda vez que de manera concomitante quienes lo cometen casi siempre son hombres.

En este estudio los efectos comprenden daños emocionales, conductuales, físicos, económicos, laborales y de estudio, que causa el hostigamiento sexual a las personas afectadas, de manera inmediata y a más largo plazo.

El análisis de los efectos indicados por las y los estudiantes que sufrieron, al menos una de las manifestaciones de hostigamiento sexual consideradas en este estudio, se basa en la respuesta de poco más de la mitad de esa población, alrededor del 49\% no marcó ninguna de las opciones de respuesta.

Como se aprecia en el gráfico 1, el enojo es porcentualmente el sentimiento más frecuente, tanto en hombres como en mujeres que han vivido hostigamiento sexual, pero las mujeres lo indicaron tres veces más que los hombres. Lo cual corrobora que el hostigamiento sexual constituye un mecanismo de control para la subordinación de las mujeres.

El gráfico permite, además, apreciar que los efectos presentan diferencias por sexo, pues la magnitud de estos es distinta entre mujeres $y$ hombres. Por ejemplo, los efectos que reportan las mujeres, en orden descendente por su importancia, luego del enojo son miedo, inseguridad, irritabilidad, vergüenza, depresión, problemas con los compañeros/compañeras de estudio, desconcentración, ansiedad, trastornos de sueño y dolor de cabeza. Los porcentajes de los efectos indicados por las mujeres varían entre $17,8 \%$ aproximadamente para el enojo hasta un $3,5 \%$ para el dolor de cabeza.

GRÁFICO 1

UNIVERSIDAD NACIONAL: EFECTOS POR EL HOSTIGAMIENTO SEXUAL SEGÚN SEXO DEL O DE LA ESTUDIANTE QUE RESPONDIERON HABERLO SUFRIDO CON BASE EN MANIFESTACIONES

(NO INCLUYE CASOS SIN RESPUESTA)

Porcentaje

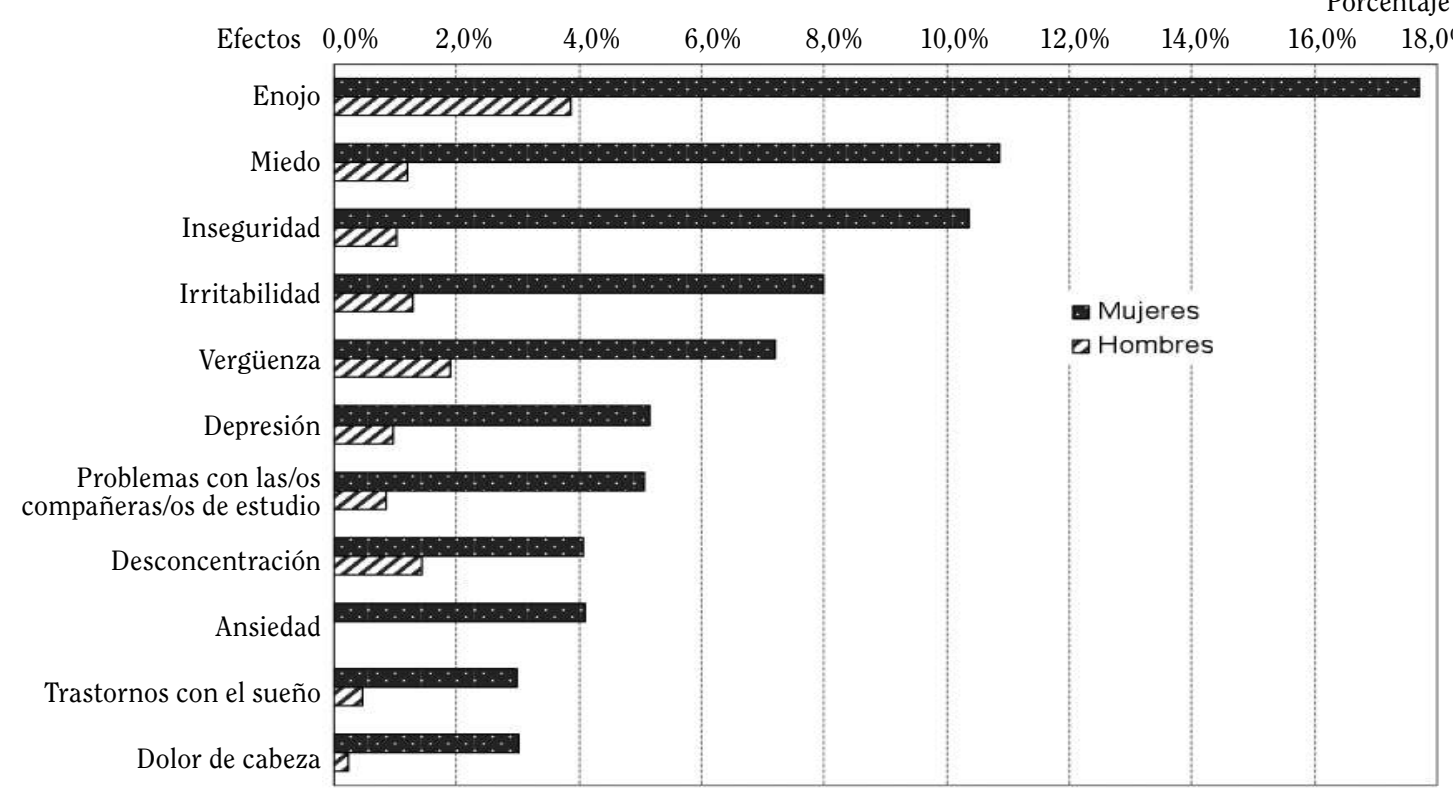


En contraposición a los efectos indicados por ellas, el orden descendente de los hombres es como sigue: enojo, vergüenza, desconcentración, irritabilidad, miedo, inseguridad, depresión, problemas con los compañeros/compañeras de estudio, de sueño y dolor de cabeza. Sin embargo, los porcentajes de respuesta de los hombres oscilan entre 3,9\% para el enojo a $0,2 \%$ para el dolor de cabeza (esto si se excluye la ansiedad, que no fue indicado por ningún estudiante como un efecto del hostigamiento sexual recibido).

En síntesis, el hostigamiento sexual es dirigido mayoritariamente contra las mujeres $y$ sus efectos son en intensidad e importancia relativa mayores que lo que les sucede a los hombres. sexual es

Según Wise y Stanley, el hostigamiento

... un método muy práctico y a menudo eficaz de hacernos guardar nuestro lugar. El propósito es reducir a las mujeres a la condición de objetos de los caprichos y deseos de los hombres y así realzar la percepción que ellos tienen de sí mismos: es en realidad como lo señaló con astucia Virginia Woolf, un medio que tienen estos hombres de mirarse en un espejo simbólico que les devuelve una imagen gigantescamente poderosa (1992: 90).

Mientras que para las mujeres violentadas por el hostigamiento sexual, los efectos son realmente dañinos, como lo expresó una estudiante entrevistada por Carvajal (2004: 81); "... me siento como que me han hecho menos de lo que yo soy...". Otra estudiante entrevistada indicó “... él logró su objetivo de herirme, traumarme...". Es obvio que estos efectos que van a perdurar en el tiempo, con la presencia de imágenes recurrentes de los hechos abusivos. Por otra parte, también queda claro que en el hostigamiento sexual no hay ninguna reciprocidad entre el hostigador y la hostigada, quien más bien lo rechaza.

No se trata, por supuesto, de minimizar los efectos que el hostigamiento sexual causa a los hombres, sino reiterar que es una práctica sistemática y discriminatoria intencionalmente dirigida a mantener a las mujeres en condiciones de opresión y subordinación a los hombres. Dicho de otra forma, como lo reflejan los datos de este estudio, los hombres no viven el hostigamiento sexual como una forma de discriminación en su contra, sino como un hecho aislado $y$ por ende, el malestar que les provoca es menor.

\section{REACCIONES}

Se debe apuntar que el análisis de reacciones se basa en poco menos de dos terceras partes de la población estudiantil (gráfico 2) que indicaron haber recibido al menos una manifestación de hostigamiento sexual, pues la otra tercera parte dejó en blanco esta pregunta.

Para la mayoría de las reacciones consideradas en este estudio, como se muestra en el gráfico 2, las mujeres son las que evidencian mayores porcentajes de respuesta.

La excepción a lo anterior, se presenta en "no le dio importancia" y "haberlo tomado a broma", donde el porcentaje de hombres supera al de mujeres que respondieron haber reaccionado de esa manera. Para los hombres como género masculino, se constata que reciben las manifestaciones del hostigamiento como un hecho aislado, es decir, más que como una acumulación intencionada para marcar rangos de inferioridad en ellos, que no corresponden en todo caso, porque se saben sujetos y no objetos sexuales $y$ por ende no es sentido como discriminación. Sobre este punto habría que exceptuar a aquellos hombres que no corresponden al estereotipo de masculinidad y que pueden ser percibidos como homosexuales. En estos casos, hay hombres que son acosados sexualmente por otros hombres heterosexuales, claramente por homofobia.

Para las reacciones consideradas en el estudio el orden descendente de importancia para las mujeres y los hombres es distinto. Que las reacciones más comunes que ellos indiquen sean que no le dio importancia y lo tomó a broma; respalda una vez más la tesis de que el hostigamiento sexual no constituye una preocupación ni es algo significativo, por las razones ya apuntadas. 
GRÁFICO 2

UNIVERSIDAD NACIONAL: REACCIONES ANTE EL HOSTIGAMIENTO SEXUAL SEGÚN SEXO DEL O DE LA ESTUDIANTE QUE RESPONDIÓ CON BASE EN MANIFESTACIONES DE HABERLO RECIBIDO

(NO INCLUYE 139 CASOS SIN RESPUESTA)

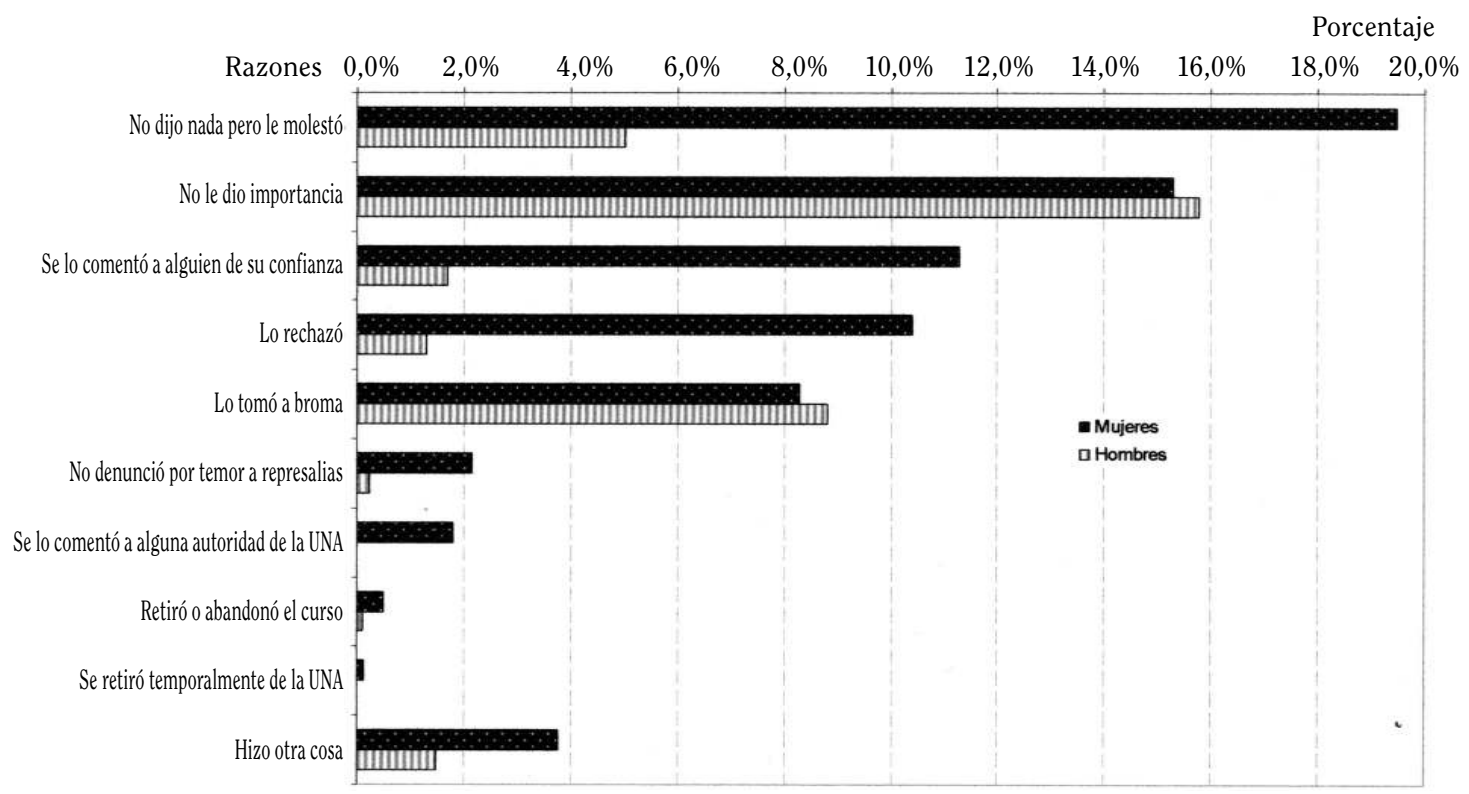

Por otra parte, llama la atención que solamente las mujeres refieren haber comentado con alguna autoridad de la UNA, aunque el porcentaje que lo indica es muy bajo.

En el contexto de una sociedad patriarcal, enfrentar el hostigamiento sexual es para las mujeres una decisión compleja. Hay que recordar que, en materia de sexualidad, la mujer siempre ha sido vista como la responsable de los hechos que se perpetran en su contra. El imaginario social todavía tiene resabios del mito de la provocación (desde Eva), por lo que las mujeres, y en particular las estudiantes, temen exponerse a ese juicio social. Como explica Maricel Salas, las "... mujeres suelen ocultar los sucesos de acoso de que son objeto por varias razones, entre ellas el temor, la vergüenza o la simple necesidad de negar la situación como un mecanismo de defensa..." (1996: 92).

Sin embargo, el silencio solo favorece la impunidad, por lo que resulta necesaria la divulgación, información, sensibilización, para hacer conciencia en la comunidad universitaria $y$ promover la denuncia.

\section{DENUNCIA Y RESPUESTA INSTITUCIONAL}

Solo el 14\% de las/los 422 estudiantes que indicaron haber recibido al menos una de las manifestaciones de hostigamiento sexual, señalan haber roto el silencio, lo cual hicieron ante personas de confianza. El porcentaje que indicó haberlo comentado a alguna autoridad de la UNA (docente, administrativo, etc.) es poco menos del $2 \%$. Conviene señalar, además, que el mismo está conformado solo por mujeres.

Sin embargo, en ninguno de estos pocos casos las y los estudiantes señalaron que le hubieran sugerido ir a la Fiscalía contra el hostigamiento sexual para interponer la denuncia, como responde según el reglamento interno.

Para que las y los estudiantes que comentaron con las autoridades, las respuestas que obtuvieron no son las que se recomiendan de acuerdo con la ley y con la reglamentación interna, lo cual es algo que como funcionarios $y$ funcionarias de la UNA, deberían dar la orientación pertinente. 


\section{GRÁFICO 3 \\ UNIVERSIDAD NACIONAL: DISTRIBUCIÓN DE ESTUDIANTES QUE RECIBIERON HOSTIGAMIENTO SEXUAL EN RELACIÓN CON ROMPER EL SILENCIO \\ NOVIEMBRE 2008}

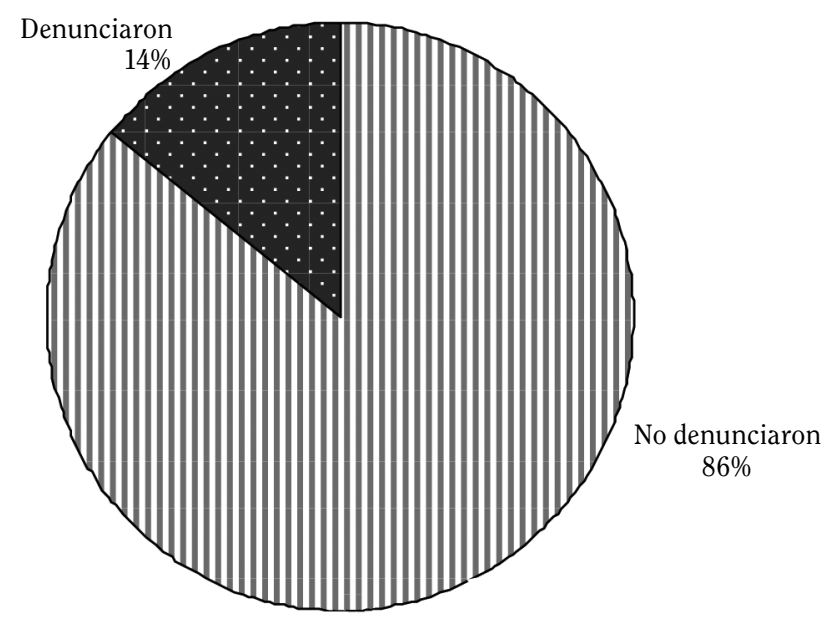

Como todo reglamento, el correspondiente a hostigamiento sexual ha sido distribuido a la comunidad universitaria por diferentes medios, pero esto pone en evidencia la necesidad de continuar con la Campaña Cero tolerancia contra el hostigamiento sexual y seguir contando con el apoyo de la Rectoría para promover cambios urgentes en la cultura institucional, que favorezcan relaciones interpersonales basadas en el respeto mutuo, la equidad entre los sexos y la igualdad de oportunidades sin ningún tipo de exclusión.

\section{CONCLUSIONES}

A partir de los hallazgos mencionados en este artículo se pueden señalar las siguientes conclusiones:

a. Los resultados de este estudio son generalizables al resto de la población estudiantil de la UNA, al basarse en una muestra aleatoria de grupos de estudiantes que se matricularon en el segundo semestre del 2008.

b. La prevalencia del hostigamiento sexual en la población estudiantil de la UNA es del 35,8\%. En términos de promedios, esto significa que una o uno de cada tres estudiantes ha sido objeto de al menos una de las manifestaciones estudiadas.

c. Las mujeres son más hostigadas que los hombres: por cada 10 hombres hostigados hay 15 mujeres hostigadas.

d. Este estudio corrobora que el hostigamiento sexual se dirige principalmente contra las mujeres, por lo que forma parte de una política sexual discriminatoria.

e. El enojo es porcentualmente el sentimiento más frecuente, tanto en hombres como en mujeres que han vivido hostigamiento sexual, pero las mujeres lo indicaron tres veces más que los hombres.

f. Existe diferencia en los efectos que se indican en razón del género, pues la importancia de ellos es distinta para mujeres y hombres.

g. Las mujeres son las que evidencian mayores porcentajes de respuesta en relación con las reacciones que tuvieron cuando fueron objetos del hostigamiento sexual. La importancia de las categorías consideradas es diferente según el sexo.

h. El $86 \%$ de las y los estudiantes que indicaron haber recibido al menos una manifestación de hostigamiento sexual no indicaron haberlo comentado con personas de su confianza, es decir, lo mantuvieron en secreto. Lo cual favorece la impunidad. 
i. La denuncia ante las instancias con competencia para investigar $y$ sancionar, no fue reportada por ninguna de las y los estudiantes que indicaron haber recibido alguna manifestación de hostigamiento sexual.

j. Las conclusiones anteriores ponen en evidencia la necesidad de mantener $y$ fortalecer programas institucionales de prevención del hostigamiento sexual, para hacer conciencia en la comunidad universitaria tendientes a desalentar este tipo de conductas y en todo caso, promover la denuncia.

\section{BIBLIOGRAFÍA CONSULTADA}

Universidad Nacional. Agenda universitaria hacia las relaciones de equidad entre hombres y mujeres. Compromisos mínimos 2005-2010. Heredia, Costa Rica.

Batres, Gioconda. Del ultraje a la esperanza. Tratamiento de las secuelas del incesto. ILANUD. Programa Regional de Capacitación, 1997.

Carvajal Orlich, Zaira. "Prevalencia, manifestaciones $y$ efectos del hostigamiento sexual en la Universidad Nacional". [Tesis de posgrado para optar por el grado de Magíster en Estudios de la Mujer]. Heredia, Costa Rica. UNA-UCR, 2004.

Centro Nacional para el Desarrollo de la Mujer y la Familia. Ley Contra el Hostigamiento Sexual en el Empleo y la Docencia 7476. Colección Documentos 1. San José, Costa Rica, 1995.

Cruz Jaimes, Guadalupe. "Criterios: periodismo independiente". Patriarcado, machismo y sexismo. 28 de mayo de 2008 . En: $<$ http://www.criterios.com/modules.php? name $=$ Noticias $\&$ file $=$ article $\&$ sid $=13891>$

Finkelhor, David. (1979). Abuso sexual al menor. Primera reimpresión. México, DF. Ed. Pax-México, 2008: 48.
García, Blanca y Bedolla, Patricia. "Las relaciones de poder y violencia vinculadas al hostigamiento sexual". Bedolla, Patricia, et ál. (Compils). Estudios de género y feminismo II. México: Distribuciones Fontamara. UNAM. Facultad de Psicología, 1993.

García-Celay, $\mathrm{M}^{\mathrm{a}}$ Luisa Montero y Nieto Navarro, Mariano. El patriarcado: una estructura invisible. En: <http://www. stopmachismo.net/marmar2.pdf $>$

Instituto Nacional de las Mujeres Inmujeres. Las mexicanas y el trabajo III. 2004. En: <http://cedoc.inmujeres.gob.mx/ documentos_download/100514.pdf>

Lagarde, Marcela. Una mirada feminista en el umbral del milenio. Heredia, Costa Rica: CEN-MUJER. Instituto de Estudios de la Mujer, Universidad Nacional, 1999.

Lagarde, Marcela. "Género y poderes". Heredia, Costa Rica. Instituto de Estudios de la Mujer. CEN-MUJER. Universidad Nacional. s.f.

Lerner, Gerda. La creación del patriarcado. Barcelona, España: Editorial Crítica, 1986.

Rich, Adrianne. Nacida de mujer. España, Barcelona. Ed. Noguer, 1978: 22.

Salas Torres, Maricel. "Los significados psicosociales del acoso sexual a las mujeres en el ambiente de trabajo: El caso del ICE". [Tesis de posgrado para optar por el grado de Magíster en Estudios de la Mujer]. MAEM. UNA-UCR, 1996.

Salas Calvo, José Manuel. "La mentira en la construcción de la masculinidad". Revista Costarricense de Psicología 24. 1996: 67-78. 
Sau, Victoria. Diccionario ideológico feminista. España, Barcelona. Icaria Editorial SA, 1989: 238-239.

Vicente, Esther. "Derecho y cambio social". Violencia contra la mujer. Un tema clave hacia Beijing 95: Desde Nairobi a Beijing 5 (o cómo se hace camino al andar). Convención Interamericana para Prevenir, Sancionar y Erradicar la Violencia contra la Mujer. Chile, Santiago. Isis Internacional, 1994: 5.
Villarreal Chávez, Nischma. "Algunas consideraciones sobre el ACOSO SEXUAL en el empleo". Módulo 4. Lectura de apoyo 1. En: <http://ns.ccp.ucr.ac.cr/ cmarin/ hostigamiento/lec41.htm>

Wise, Sue y Stanley, Liz. El acoso sexual en la vida cotidiana. Barcelona. Ediciones Piadós, 1992: 90. 